\title{
Development of a Finite Element Analysis Program for Roller Leveling and Application for Removing Blanking Bow Defects of Thin Steel Sheet
}

\author{
Kee-cheol PARK and Sang-Moo HWANG ${ }^{11}$
}

Sheet Products and Process Research Group, Technical Research Labs., POSCO, Goedong-dong, Nam-gu, Pohang-shi, Gyungbuk, 790-785, Korea. E-mail: parkkc@posco.co.kr 1 1) Department of Mechanical Engineering, Pohang University of Science \& Technology, San 31, Hyoja-dong, Nam-gu, Pohang-shi, Gyungbuk, 790-784, Korea.

(Received on Febuary 18, 2002; accepted in final form on May 28, 2002)

\begin{abstract}
For the analysis of roller leveling process and application for controlling blanking bow defects in a thin steel sheet, a finite element analysis program modeling large deformation of shell has been developed. This program for elastic-plastic large deformation analysis of sheet includes spring-back analysis as well as contact treatment between sheet and rolls of roller leveler. This is verified by the simple leveling experiment with 5 rolls at laboratory. The residual curvatures of strip predicted by finite element analysis are within $20 \%$ error range of the experiment. An optimum entrance intermesh condition, that is able to remove the blanking bow or deviation of curvature of a sheet, are found by finite element analysis for initially curved three strips by applying virtually divided strip model. The curvature difference of strips after leveling is decreased as increasing the entrance intermesh of roller leveler, and the optimum intermesh value is affected by the roll diameter and arrangement. The predicted optimum intermesh values are consistent with the experimental results. The residual stress distributions after leveling is predicted numerically by the curvature of strip obtained from the finite element analysis. It is found that the change in the residual stress distributions due to leveling is also markedly affected by the intermesh condition.
\end{abstract}

KEY WORDS: blanking bow; spring-back; finite element analysis; residual stress; roller leveling; intermesh.

\section{Introduction}

Sheet steel has various shape defects, such as edge wave, center buckle, curl, gutter, and blanking and etching bow as shown in Fig. 1(a). ${ }^{1)}$ Edge wave and center buckle in sheet are caused by difference in length of sheet between center and edges, while curl and gutter are due to the moment developed by the distribution of in-plane normal stress component along the thickness. These shape defects could be easily found from sheet state before blanking or etching. But, in general, blanking bow and etching bow are appeared after blanking or chemical etching, only. ${ }^{2)}$ Etching bow is due to the non-uniform distribution of residual in-plane normal stress component along thickness direction, and blanking bow is due to the non-uniform distribution of moment, induced by the in-plane normal stress component along thickness, at each position in the same sheet. Blanking and etching bow cause problems in stacking of blanks as illustrated in Fig. 1(b) as well as feeding to forming or painting machines in the 3-piece can making and shadow mask processes. ${ }^{2)}$

The effectiveness of roller leveler on the bow (curl and gutter), blanking and etching bow are well known, ${ }^{1,3)}$ because it is a device for flattening sheet as well as reducing magnitude of residual stress by using monotonically decreasing reverse bending due to arrangement of rolls and the associated plastic deformation of sheet. The entrance and exit intermesh of upper and lower roll sets control the degree of monotonically decreasing repeated bending. The

\begin{tabular}{|c|c|c|c|c|c|}
\hline \multicolumn{2}{|c|}{ Length difference } & \multicolumn{2}{|c|}{ Curvature } & \multicolumn{2}{|c|}{ Curvature after forming } \\
\hline $\begin{array}{l}\text { Edge } \\
\text { wave }\end{array}$ & $\begin{array}{l}\text { Center } \\
\text { buckle }\end{array}$ & Curl & Gutter & $\begin{array}{l}\text { Blanking } \\
\text { Bow }\end{array}$ & $\begin{array}{l}\text { Etching } \\
\text { Bow }\end{array}$ \\
\hline & 0000 & & & $\rightleftarrows$ & 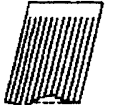 \\
\hline
\end{tabular}

(a) Shape defects of sheet $^{1)}$



(b) Banking bow

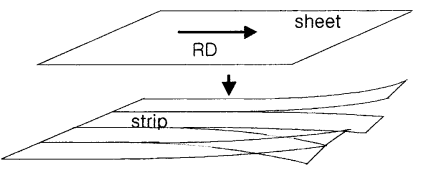

(c) Virtually divided strip model for blanking bow
Fig. 1. Shape defects of sheet and virtually divided strip model for blanking bow. 
role of the entrance intermesh is reducing the curvature (in rolling direction) deviation of leveled sheet, and the exit intermesh is to adjust average curvature to zero. ${ }^{3)}$ Therefore, the major problem in roller leveling process to be found is the entrance intermesh value that is sufficient for removing curvature deviation within the incoming sheet. And, the curvature deviation within a leveled sheet is mainly due to improper condition of entrance intermesh values of leveler for removing the deviation of curvature in incoming sheet at each length and width position.

In the case of tension leveling, there have been some attempts in order to determine the optimal process condition for leveling. In these works, in order to predict the curvature of strip during leveling process, both experimental and analytical models are presented. In the experimental models, ${ }^{4-6)}$ the curvature of strip is explicitly defined by the mechanical properties, thickness of the strip, the roll intermesh, the roll pitch, and the applied tension. On the other hand, some simplified analytical models ${ }^{7,8)}$ have also been presented, but only a few are based on finite element analysis. Yoshida and Urabe ${ }^{9)}$ proposed a computer aided process design based on the finite element method. They used the steady finite element analysis and the constitutive model of cyclic elastic-plasticity to describe accurately the cyclic behavior of strips. In order to determine the optimal process condition for roller leveling, there have been few attempts. $\mathrm{Kano}^{10)}$ recently analyzed roller leveling by using finite element method. His formulation considered large deformation of sheet and treated contact conditions by iteratively to meet geometric constraint. Strip was modeled by using 6 layers of 4-node plane strain element with selective reduced integration, and rolls were treated as rigid segments. He compared the curvature of strip during leveling and residual curvature obtained from finite element analysis to the strip curvature obtained from Misaka ${ }^{4)}$ model, and showed that that model is not proper for roller leveling analysis. But modeling of thin sheet by continuum element requires large number of element and there are some problems in treating plastic bending accurately.

In order to find the tension leveling condition for wavy sheet, Hira ${ }^{11)}$ used virtually divided strip model for the sheet with different initial length for each divided strip according to the initial steepness of sheet. This virtually divided strip model assumes that the interaction between divided strips is negligible. In the case of roller leveling, applied tension is negligibly lower than tension leveling and the major deformation mode is only bending, the interaction between divided strips is less than tension leveling. Therefore, this model will be useful for investigating the optimum condition of roller leveler for removing blanking bow.

In this study for analyzing the optimum condition for blanking bow control by roller leveler, the virtually divided strip model is adopted. In the procedure, a sheet before leveling is slit into several segments as shown in Fig. 1(c), to measure the curvature of each segment. Then, investigated is the deformation of three segments-those showing the maximum curvature either upward or downward, and also a segment showing an intermediate value-during leveling. After leveling, the differences of residual curvature between leveled strips are used as the measure of blanking bow de- fects. As a tool for analyzing these processes, such as flattening initially curved strip, leveling and spring-back, effectively, a finite element program has been developed. This program for elastic-plastic large deformation analysis of sheet during leveling includes spring-back algorithm as well as special contact treatment between sheet and rolls and integration of stress along thickness direction.

After a brief introduction of finite element procedure, some verification and analysis results are followed. In order to apply the developed roller leveling analysis program for blanking bow problems, three virtually divided plane-strain strips with different initial curvatures are analyzed at different entrance intermesh values. The optimum intermesh values for removing the curvature deviation of three sheets to zero are found and the results are verified by the experiment. Furthermore, the effect of roller leveling conditions on residual stress are analyzed and proved by experiment.

\section{Analytical Procedures for Modeling Roller Leveling Process}

\subsection{Description of Geometry}

The strip is modeled by the 3-dimensional shell element for accurate description of bending effect. However, the problems analyzed in this paper may be assumed as plane strain, so that one coordinate direction is constrained by boundary conditions. The geometry and displacement of 4node shell element are described by the method generally used in degenerated shells such as Hughes. ${ }^{12)}$ The mapping between reference and real coordinate is described in Fig. 2. The geometry of a typical shell element is defined by using the position vector of a point in the middle surface, which is defined by two-dimensional shape functions (for $\xi$ and $\eta$ ) and associated nodal point coordinates, and a position vector based at a point in the middle surface that defines the 'fiber direction $(\zeta)$ ' through the point.

Lamina surface is defined as $\zeta$ fixed, and fiber direction is the line defined as $\xi, \eta$ fixed. The fibers are not generally perpendicular to the lamina surface. The kinematics of the shell element is also defined by invoking the iso-parametric hypothesis. The displacement vector of a generic point of the shell is defined by the displacement of a point on the middle surface, which is interpolated from the displacement vector of the nodal point, and the fiber displacement, which is computed from the displacement of unit fiber vector. The number of nodal variables (degree of freedom) of a node in shell elements in global coordinate system was six, three displacement and 3 rotation increments. The rotation increment vector in global coordinate system $\left(\Delta \theta_{\mathrm{a}}\right)$ is related with the displacement of the unit fiber vector through vector product with the unit fiber vector.

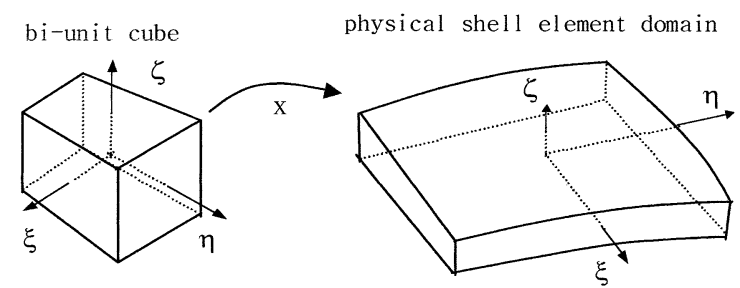

Fig. 2. Smooth mapping of the bi-unit cube into the physical shell domain. 


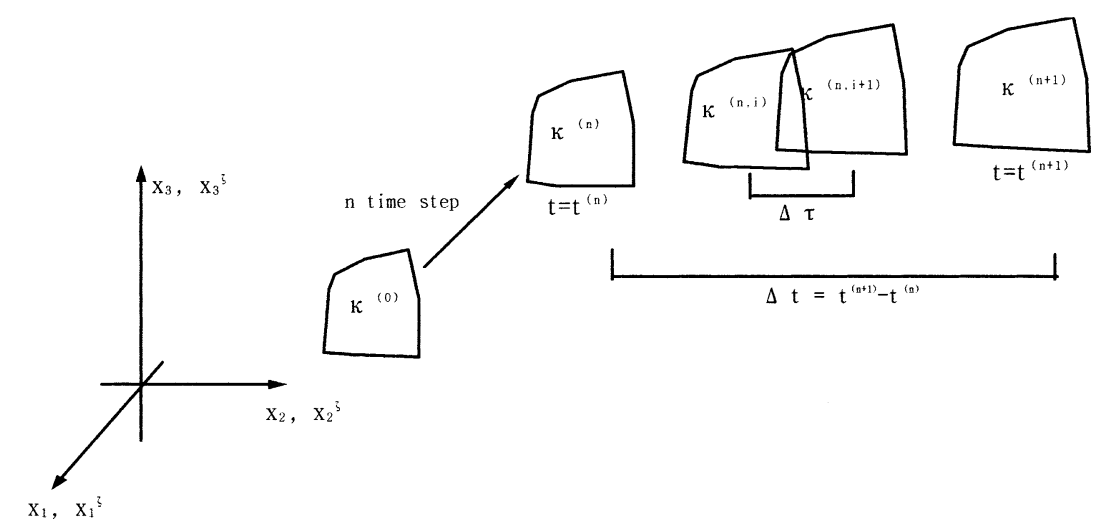

Fig. 3. Un-deformed and updated reference configuration at incremental deformation calculation.

\subsection{Constitutive Equations}

Elastic-plastic rate constitutive equations are used in this research. Considering a rate type constitutive equation regarding large deformation for Prantl-Reuss theory, ${ }^{13-15)}$ the relationship between the stress rate and strain rate can be represented as following Eq. (1).

$$
\dot{\tau}_{i j}^{\mathrm{J}}=C_{i j k l} D_{k l}
$$

$\dot{\tau}_{i j}^{\mathrm{J}}$ is the Jaumann derivative of Kirchhoff stress, and $D_{k l}$ is the rate of deformation. Kirchhoff stress is introduced to obtain symmetric tangent stiffness matrix, ${ }^{13,16)}$ but Kirchhoff and Cauchy stress are the same if the reference state is assumed as present state. In this paper, since updated Lagrangian formulation ${ }^{13)}$ is adopted, Kirchhoff and Cauchy stress are considered as the same.

In developing a discrete algorithm based upon above rate type constitutive equation, the Eq. (1) is written with respect to the lamina coordinate system at time $t^{(n+1)}$. Furthermore, the stress components at time $t^{(n)}$ are defined with respect to the $t^{(n)}$ lamina system and that $\sigma_{33}^{n}=0$. The stress components at time $t^{(n)}$ is transformed by the rotation vector of the material particle relative to the laminar basis and then the co-rotational stress increments are computed from Eq. (1), as in Eq. (3).

$$
\begin{array}{rlr}
\bar{\sigma}^{(n+1)}=R \sigma^{(n)} R^{T} & \text { where } & R=e^{W \Delta t} Q \ldots \ldots \ldots \ldots . . . \\
\sigma^{(n+1)}=\bar{\sigma}^{(n+1)}+\Delta \sigma & \text { where } \quad \Delta \sigma_{i j}=C_{i j k l} \Delta \varepsilon_{k l} \ldots \ldots .
\end{array}
$$

in which $W$ is the spin tensor, $\Delta t$ is the time increment and $Q$ is the incremental transformation tensor between lamina bases (from lamina at $t^{(n)}$ to lamina at $t^{(n+1)}$ ). This algorithm is the same as Hughes ${ }^{17)}$ and it is incrementally objective for laminar system that is rotating with the mid plane of the sheet. For imposing the plane stress condition for shell element, Eq. (4) is also used.

$$
\Delta \varepsilon_{33}=-\left(\bar{\sigma}_{33}^{(n+1)}+\sum_{i j \neq 33} C_{33 i j} \Delta \varepsilon_{i j}\right) / C_{333}
$$

Under the Mises yield criterion and the associated flow rule, return mapping ${ }^{18,19)}$ algorithms (elastic predictor radial return) are used as integration scheme, and the consistent tangent operator to this nonlinear incremental model derived by $\mathrm{Simo}^{18,19)}$ is used for improving convergence of Newton scheme.

\subsection{Finite Element Model for the Analysis of the Non- steady Plastic Deformation of the Sheet Material}

The non-steady state finite element analysis is carried out in order to analyze the effect of deformation due to the initial curvature and processing on the spring back phenomenon. Updated Lagrangian formulation ${ }^{13)}$ is adopted, since the elastic-plastic constitutive equation that treats incremental displacement based on the present state is used. Considering a deforming work piece $V$ with the traction $\bar{t}_{i}$ prescribed on $S_{t_{i}}$ of the surface $S_{t}$ and the displacement $u_{i}=\bar{u}_{i}$ prescribed on $S_{u_{i}}$ of the surface $S_{u}$ except the contact interface. Let $S_{\mathrm{c}}$ be the remainder of the surface and assume that $S_{\text {c }}$ represents the roll-sheet contacting interface. The friction in the contacting surface between sheet and roll is ignored by assuming that friction might be negligible. As described in Fig. 3, after just finished up to the $i$-th iteration of $n$-th time step or the iteration cycle $(n, i)$, to solve for time step $t=t^{(n+1)}$, the governing equation, with neglecting body force and inertia effects, for $(n, i+1)$ with reference to the fictitious configuration $(n, i)$ is set up as follows.

$$
\begin{aligned}
& \frac{\partial T_{i j}^{\zeta(n, i+1)}}{\partial x_{i}^{\varsigma}}=0 \text { in } V^{\zeta} \\
& T_{i j}^{\zeta(n, i+1)} n_{i}^{\zeta}=\bar{t}_{j} \quad \text { on } \quad S_{t_{j}}^{\zeta} \\
& u_{i}^{(n+1)}=\bar{u}_{i}^{(n+1)} \quad \text { on } \quad S_{u_{i}}^{\zeta} \\
& u_{i}^{(n, i+1)} n_{m_{i}}^{\zeta}=\bar{u}_{i}^{(n+1)} n_{m_{i}}^{\zeta} \quad \text { on } \quad S_{c_{m}}^{\zeta} \text { where } \zeta=t^{(n, i)}
\end{aligned}
$$

The reference state $\zeta=t^{(n, i)}$ is some fictitious time unless the corresponding configuration $\kappa^{(n, i)}$ is an equilibrium configuration. $T_{i j}^{\zeta}$ is the nominal stress (1st Piola-Kirchhoff stress) based on $t=\zeta, n_{i}^{\zeta}$ is the unit normal vector of the surface $S_{t_{i}}^{\zeta}$, and $n_{m_{i}}^{\zeta}$ is the unit normal vector of contacting master (roll) surface. Traction and displacement boundary conditions are imposed as boundary conditions.

The roll (master) surfaces are modeled by finite elements, due to its relative consistent treatment of contact between sheet and rolls. The algorithm proposed by Benson ${ }^{20)}$ is adopted for contact search. An interface model for thin shells reported by Zhong ${ }^{21)}$ is adapted for incorporating the fact that the contact only occurs on the physical boundary of a shell element, not on its reference surface or mid plane. Regarding the evaluation of the nodal contact force, the normal vector drawn normal to the roll surface at contact- 
ing point (which is $n_{m}$ in Eq. (6)) is used when evaluating its magnitude, while the normal vector drawn normal to the sheet at contacting node (which is $n_{j}$ in Eq. (6)) is used when evaluating its direction. Such a mixed treatment of a normal vector is adopted to improve the convergence characteristics of a contact problem. ${ }^{20,22)}$ Then, the force applied on the contacting slave node can be obtained as in Eq. (6).

$$
\begin{aligned}
F_{c_{j}}^{(n, i+1)} & =n_{j}^{\varsigma} k\left\{\left(\bar{u}^{(n+1)}-u_{i}^{(n, i+1)}\right) n_{m_{i}}^{\varsigma}\right\} \\
& =n_{j}^{\varsigma} k\left\{\left(\bar{x}_{i}^{(n+1)}(\xi, \eta)-x_{i}^{(n, i+1)}\right) n_{m_{i}}^{\varsigma}-\frac{h}{2}\right\}
\end{aligned}
$$

Where $k$ is the contact stiffness coefficient or penalty parameter, the reference state $\zeta=t^{(n, i)}$, and $h$ is the sheet thickness.

By applying stress increment equation, $T^{\zeta(n, i+1)}=T^{\zeta(n, i)}+$ $\Delta T^{\zeta(n, i+1)}$, and the divergence theorem to weak form of governing equation, the following relation is obtained for all kinematical admissible displacement $w_{j}$ where $\zeta=t^{(n, i)}$.

$$
\begin{array}{r}
\int_{V^{\varsigma}} \frac{\partial w_{j}}{\partial x_{i}^{\varsigma}} \Delta T_{i j}^{\zeta(n, i+1)} d V+\int_{S_{c_{i}}^{\varsigma}} w_{n} k \Delta u_{n}^{(n, i+1)} d S=\int_{S_{t_{j}}^{\varsigma}} w_{j} \bar{t}_{j}^{(n+1)} d S \\
\quad+\int_{V^{\varsigma}} \frac{\partial w_{j}}{\partial x_{i}^{\varsigma}} T_{i j}^{\varsigma(n, i)} d V+\int_{S_{c_{i}}^{\varsigma}} w_{j} n_{j}^{\varsigma} k\left(\bar{u}_{i}^{(n+1)}-u_{i}^{(n, i)}\right) n_{m_{i}}^{\varsigma} d S
\end{array}
$$

For incorporating constitutive equations, the following relation $^{13,16)}$ with the objective (Jaumann) increment of stress are introduced. This equation is true in the case that reference state is the same as present state,

$$
\begin{aligned}
\Delta T_{i j}^{\zeta(n, i+1)}= & \Delta \tau_{i j}^{J(n, i+1)}-\Delta \varepsilon_{i m}^{(n, j+1)} \sigma_{m j}^{(n, i)} \\
& -\sigma_{i m}^{(n, i)} \Delta \varepsilon_{m j}^{(n, i+1)}+\sigma_{i m}^{(n, i)} \frac{\partial \Delta u_{j}^{(n, i+1)}}{\partial x_{m}^{\varsigma}} \ldots . .
\end{aligned}
$$

Substituting Eq. (8) into Eq. (7), and considering a tangent modulus consistent with the radial return algorithm, ${ }^{18,19)} C^{(n, i)}$, the finite element procedure are formulated as Eq. (9).

$$
\begin{gathered}
\int_{V^{\zeta}} \varepsilon_{i j}^{*} C_{i j k l}^{(n, i)} \Delta \varepsilon_{k l}^{(n, i+1)} d V-\int_{V^{\varsigma}}\left\{\Delta \varepsilon_{i m}^{(n, i+1)} \tau_{m j}^{(n, i)}+\tau_{i m}^{(n, i)} \Delta \varepsilon_{m_{j}}^{(n, i+1)}\right. \\
\left.-\tau_{i m}^{(n, i)} \frac{\partial \Delta u_{j}^{(n, i+1)}}{\partial x_{m}^{\varsigma}}\right\} \frac{\partial w_{j}}{\partial x^{\varsigma}} d V+\int_{c_{i}} w_{j} n_{j} k n_{m_{i}} \Delta u_{i}^{(n, i+1)} d S \\
=\int_{S_{t_{j}}^{\varsigma}} w_{j} \bar{t}_{j}^{(n+1)} d S-\int_{V^{\varsigma}} \frac{\partial w_{j}}{\partial x_{i}^{\varsigma}} \sigma_{i j}^{(n, i)} d V+\int_{S_{c_{i}}^{\varsigma}} w_{j} n_{j} k\left(\bar{u}_{i}^{(n+1)}-u_{i}^{(n, i)}\right) n_{m_{i}} d S
\end{gathered}
$$

The element under consideration is the 4-node bilinear quadrilateral. For overcoming locking problems in shell element, selective reduced integration ${ }^{12)}$ for transverse shear strain term is used.

\subsection{Spring-back Analysis}

At the end of the leveling process, the external force due to tractions and contact boundaries is given as Eq. (10).

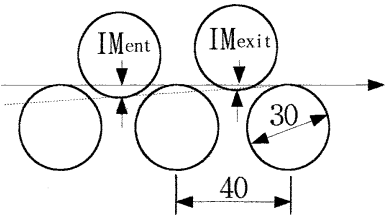

Fig. 4. Experimental roller leveler used for verification.

Table 1. Mechanical properties of tested sheet used for verification experiment.

\begin{tabular}{|c|c|c|c|c|c|c|}
\hline Sheet & Thick $(\mathrm{mm})$ & YS $(\mathrm{MPa})$ & $\mathrm{YP}-\mathrm{E} 1 .(\%)$ & TS $(\mathrm{MPa})$ & $\mathrm{U}-\mathrm{E} 1 .(\%)$ & T-El.(\%) \\
\hline A & 0.21 & 368 & 0.6 & 447 & 13.9 & 22.2 \\
\hline B & 0.23 & 222 & 0.0 & 358 & 19.9 & 30.5 \\
\hline C & 0.30 & 196 & 0.0 & 355 & 24.4 & 43.1 \\
\hline D & 0.30 & 310 & 2.1 & 397 & 20.4 & 32.7 \\
\hline
\end{tabular}

$$
A=\int_{S_{t_{j}}^{\varsigma}} w_{j} \bar{t}_{j}^{f} d S+\int_{S_{c_{i}}^{\varsigma}} w_{j} n_{j} k\left(\bar{u}_{i}^{f}-u_{i}^{f}\right) n_{m_{i}} d S
$$

Where the superscript $f$ represents the final leveling time step. Note that the values of all the terms having ' $f$ ' can be obtained from simulation.

During unloading, the final external force, described by Eq. (10), should be reduced to zero. Assuming that unloading is performed gradually, a parameter $\alpha$ is introduced, as follows:

$$
\begin{gathered}
\int_{V^{\varsigma}} \varepsilon_{i j}^{*} C_{i j k l}^{(n, i)} \Delta \varepsilon_{k l}^{(n, i+1)} d V-\int_{V^{\varsigma}}\left\{\Delta \varepsilon_{i m}^{(n, i+1)} \tau_{m j}^{(n, i)}+\tau_{i m}^{(n, i)} \Delta \varepsilon_{m j}^{(n, i+1)}\right. \\
\left.-\tau_{i m}^{(n, i)} \frac{\partial \Delta u_{j}^{(n, i+1)}}{\partial x_{m}^{\varsigma}}\right\} \frac{\partial w_{j}}{\partial x_{i}^{\varsigma}} d V=(1-\alpha) A-\int_{V^{\varsigma}} \frac{\partial w_{j}}{\partial x_{i}^{\varsigma}} \sigma_{i j}^{(n, i)} d V
\end{gathered}
$$

Note that, when $\alpha=1$, the spring back solution is achieved. Starting from $\alpha=0, \alpha$ is increased by 0.2 at each unloading step, which take five steps to obtain the springback solution. The gradual unloading strategy is adopted in the present investigation, since one step unloading strategy often fails to achieve solution convergence, especially when the elastic recovery involves a large rotation.

\section{Verification with Leveling Experiment for Residual Curvature and Residual Stress}

In order to verify the finite element procedure implemented in this paper, a simple leveling experiments are conducted at laboratory. The number of rolls in roller leveler is five and the axes of roll are supported by bearings to minimize frictional effect. Schematic illustration of the test apparatus and experimental conditions are shown in Fig. 4. Experiments are done by 4 kinds of sheets at two different intermesh values. The mechanical properties of specimens are listed in Table $\mathbf{1}$ and the stress-strain relations around yield stress are shown in Fig. 5. Specimen length is $620 \mathrm{~mm}$ and width is $50 \mathrm{~mm}$. After clamping in the roller leveler at given intermesh values, the strip is drawn to $350 \mathrm{~mm}$ length. The deformation of sheet reaches steady state condition after drawing $150 \mathrm{~mm}$ length, and the curvature of sheet is computed by coordinates of three points measured within further $200 \mathrm{~mm}$ length drawn part. For finite element 

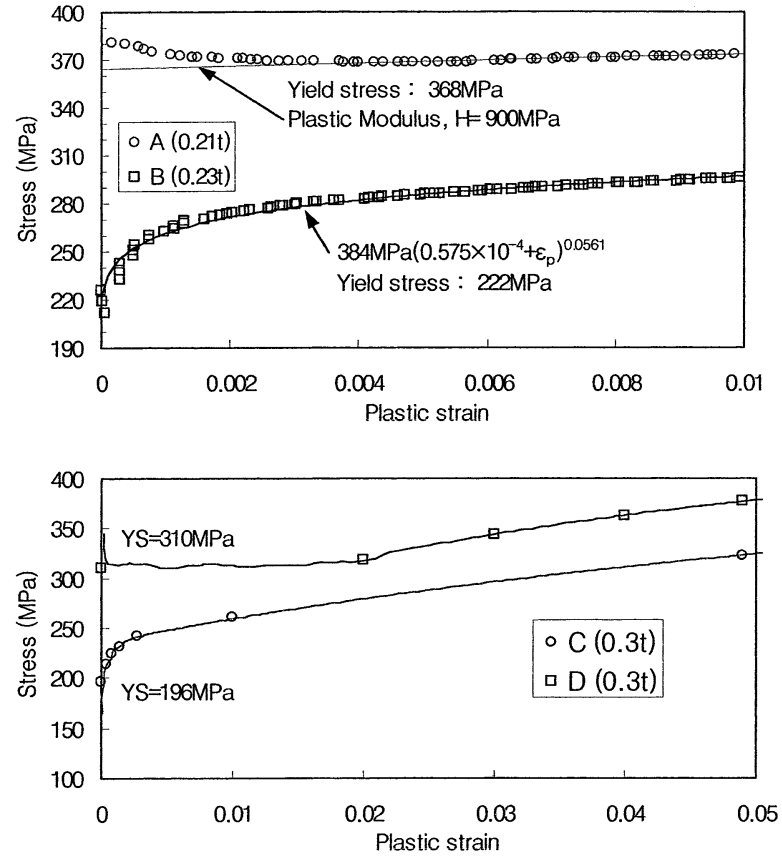

Fig. 5. True stress-plastic strain relation near yield stress for tested sheets.

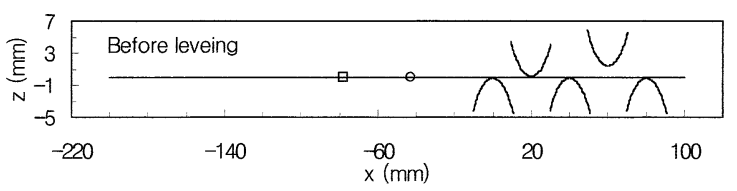

(a) Before leveling process

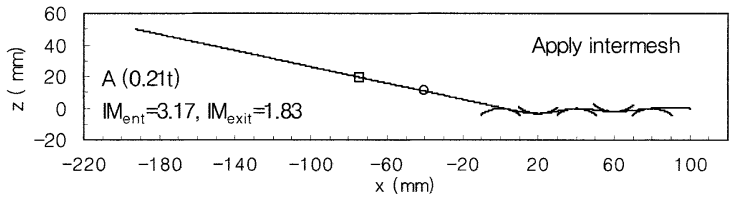

(b) Apply intermesh

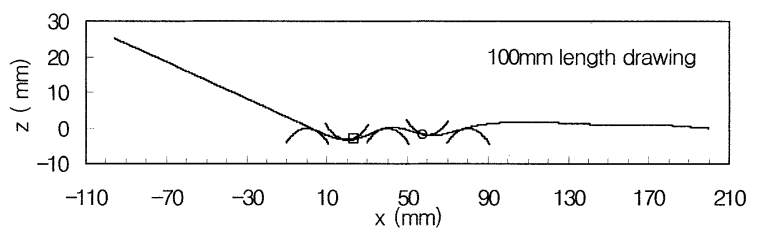

(c) After $100 \mathrm{~mm}$ length drawing

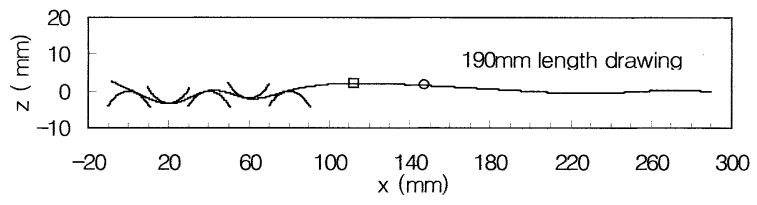

(d) After $190 \mathrm{~mm}$ length drawing

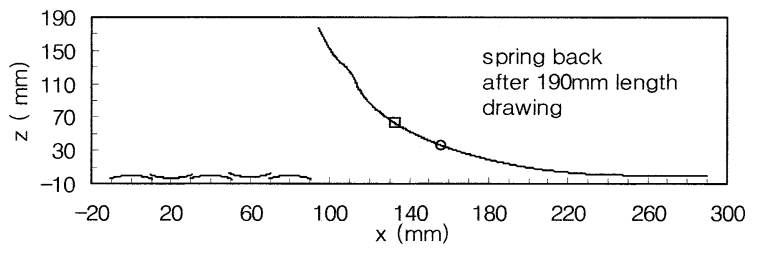

(e) Spring back by removing constraint of rolls

Fig. 6. Procedures of roller leveling process simulation for verification.

simulation, sheet is modeled by shell element with $0.5 \mathrm{~mm}$ length and total length of sheet modeled was $300 \mathrm{~mm}$. Rolls are modeled by $0.47 \mathrm{~mm}$ segment length. Number of inte-
Table 2. Curvatures of strip, deformed at steady state condition, after spring back.

\begin{tabular}{|c|c|c|c|c|c|c|c|c|}
\hline \multirow[b]{2}{*}{ Tested sheets } & \multirow{2}{*}{\multicolumn{2}{|c|}{$\begin{array}{c}\text { A } \\
\text { Thick. }=0.21 \mathrm{~mm} \\
Y \mathrm{~S}=368 \mathrm{Mpa},\end{array}$}} & \multicolumn{2}{|c|}{ B } & \multicolumn{2}{|c|}{$\mathrm{C}$} & \multicolumn{2}{|c|}{ D } \\
\hline & & & $\begin{array}{r}\text { Thick. } \\
\text { YS= }\end{array}$ & $\begin{array}{l}.23 \mathrm{~mm} \\
2 \mathrm{MPa}\end{array}$ & $\begin{array}{l}\text { Thick. } \\
\text { YS= }\end{array}$ & $30 \mathrm{~mm}$ & $\begin{array}{r}\text { Thick }= \\
\text { YS }=\end{array}$ & \\
\hline$\underbrace{\text { Curvature }\left(\mathrm{m}^{-1}\right)}_{\text {Intermesh }(\mathrm{mm})}$ & Exp. & Cal. & Exp. & Cal. & Exp. & Cal. & Exp. & Cal. \\
\hline$I M_{\text {ent }}=1.58, I M_{\text {exit }}=0.92$ & 0.44 & 0.13 & 4.81 & 3.45 & 4.06 & 5.03 & 3.73 & 4.57 \\
\hline$I M_{\text {ent }}=3.17, I M_{\text {exit }}=1.83$ & 6.02 & 7.30 & 5.90 & 5.90 & 2.91 & 2.56 & 3.61 & 3.62 \\
\hline
\end{tabular}

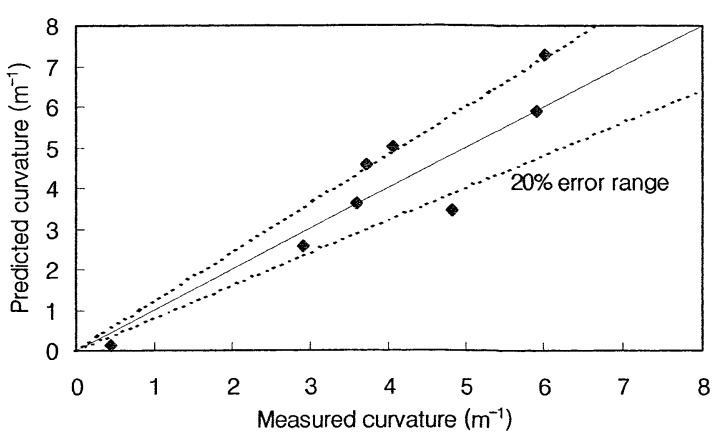

Fig. 7. Comparison of experimentally measured and predicted curvature.

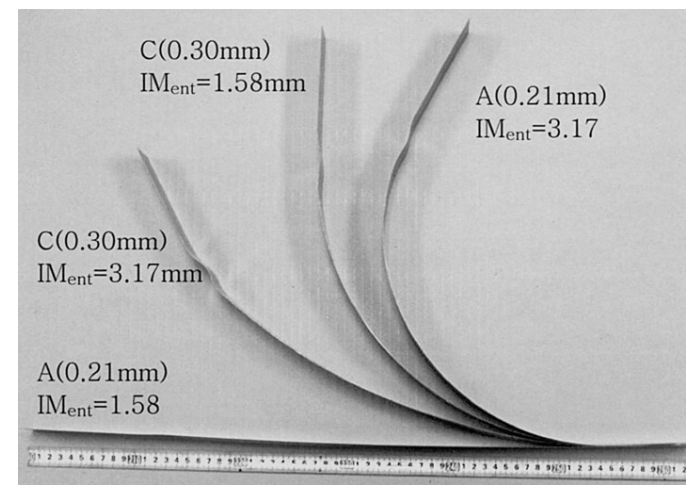

Fig. 8. Unloaded sheet after $350 \mathrm{~mm}$ drawing of $\mathrm{A}$ and $\mathrm{C}$ sheet (620 mm length) specimen.

gration point in thickness direction for shell element is nine for accurate consideration of plastic deformation. Figure 6 presents simulation processes for A-sheet at entrance and exit intermesh values of $3.17 \mathrm{~mm}$, and $1.83 \mathrm{~mm}$, respectively: clamping of sheet in the roller leveler, drawing to 190 mm length with no applied back tension and spring-back. The $35 \mathrm{~mm}$ length part of the sheet between rectangular and circle mark in Fig. 6 is used for residual curvature computation after spring back. These simulation results are compared with experimental results by the curvature of steadily deformed part of the sheet in Table 2 and Fig. 7. Predicted residual curvatures by finite element method are within $20 \%$ error range of the curvature measured by experiment. Figure 8 is the shape of sheet unloaded after drawing 350 mm length, and Fig. 9 is the predicted strip shape unloaded after $190 \mathrm{~mm}$ drawing for two sheet, A and C, for two different intermesh conditions. The strip shape and curvature between experiment and simulation has almost the same tendency for all testing conditions. Though there is some difference between experiment and simulated results, the results are not bad if the difficulty in the experiment and the measurement of spring-back with thin sheet is considered.

For computing the residual (in-plane, length directional 


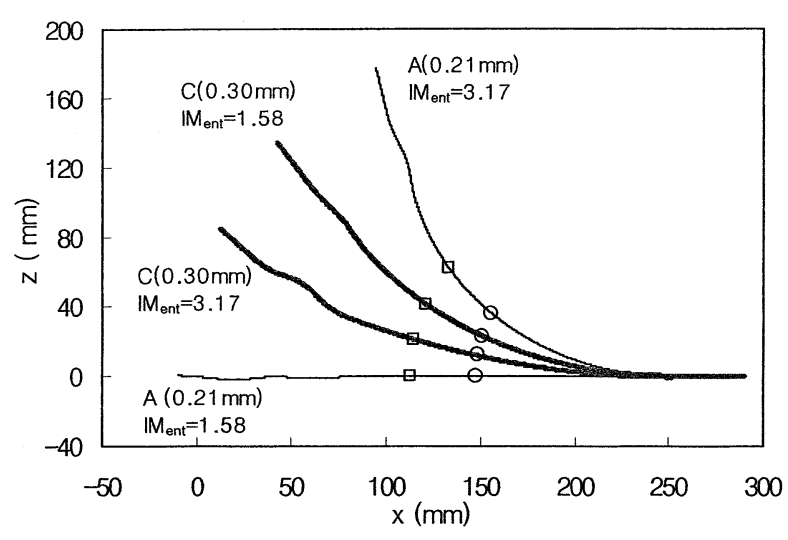

Fig. 9. Unloaded sheet after $190 \mathrm{~mm}$ drawing of $300 \mathrm{~mm}$ length $\mathrm{A}$ and $\mathrm{C}$ sheet by finite element simulation.

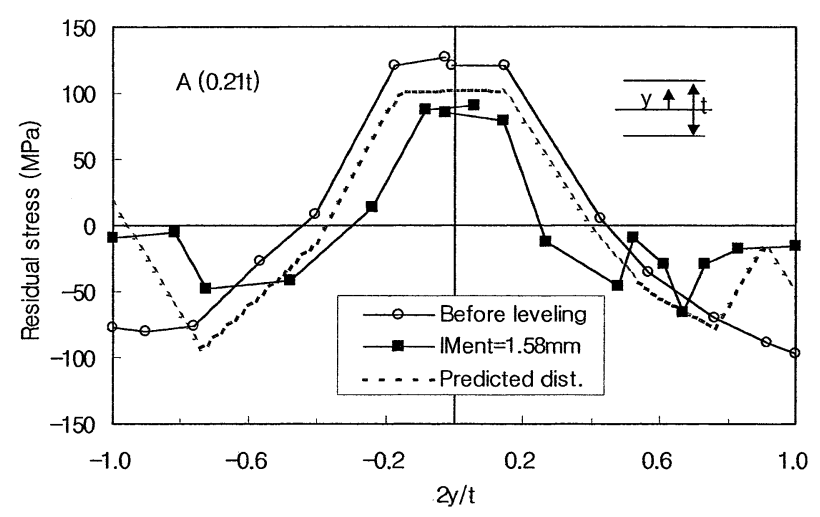

Fig. 10. Measured residual stress distribution along thickness of sheet before and after leveling, and predicted residual stress after leveling.

normal) stress distribution along thickness, in detail, Roberts ${ }^{23)}$ method with the computed curvature at each roll from the sheet shape predicted by finite element method is used. Kano ${ }^{10)}$ also predicted residual stress of leveled sheet by the same procedure. The residual stress distribution of incoming sheet and leveled sheet are measured experimentally by the surface removing method ${ }^{24)}$ for verifying the analysis results.

Figure 10 presents the experimentally measured residual stress before and after leveling process, and the residual stress distribution predicted by the curvature obtained from the finite element simulation. The simulated and experimental residual stress distributions are nearly the same, and it is possible to say that the prediction of residual stress change during leveling process by computed curvature of sheet during leveling is possible with reasonable accuracy.

\section{Analysis of Roller Leveling Process and Optimization of Operating Conditions for Removing Blanking Bow}

\subsection{Roller Leveling Process Modeling for Controlling Blanking Bow}

Two types of roller leveler in real production lines, described in Fig. 11, are analyzed, one roller leveler has 13 rolls of $68 \mathrm{~mm}$ diameter and the other has 15 rolls of $58 \mathrm{~mm}$ diameter. In roller leveling technology, one of the most important problem is "what is the entrance intermesh value

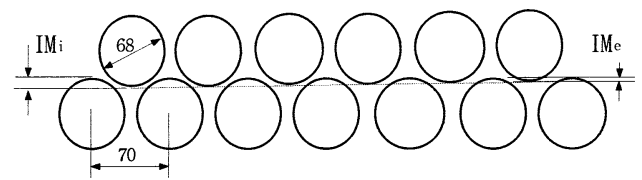

(a) A-Line roller leveler

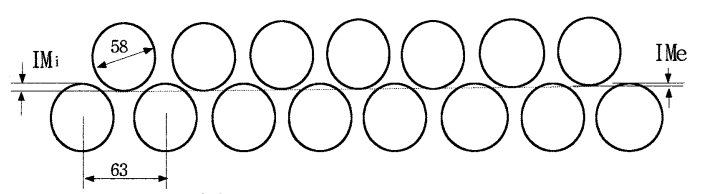

(b) B-Line roller leveler

Fig. 11. Roller levelers considered.

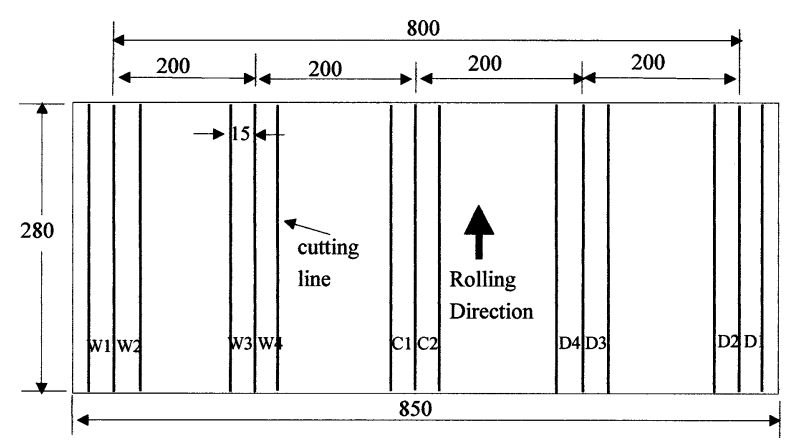

Fig. 12. Cutting line in a sheet for measuring curvature distribution.

that is suitable for removing curvature deviation of incoming sheet", because the exit intermesh is generally adjusted from near zero value to optimum level during operation for setting average curvature of leveled sheet to be flat.

Since the analysis program is formulated for 3-dimensional problems, proper boundary conditions are imposed for plane-strain deformation of strip, which is one of the virtually divided segments of a sheet. Note that, when using the virtually divided strip model, a sheet before leveling is slit into several segments having different curvatures, as shown in Fig. 1(c), and investigated is the variation of the curvature of each segment during leveling. A segment (or a strip) with a specific curvature is assumed to have enough length for obtaining steady-state deformation region after drawing. Modeled strip length is $1100 \mathrm{~mm}$ and element number is 2000 with $0.55 \mathrm{~mm}$ length of element. The measured curvature of each strip after slitting is considered as the initial curvature of each strip. Rolls, considered as rigid, are modeled only in contacting region with strip and length of roll element is $0.91 \mathrm{~mm}$ for $58 \mathrm{~mm}$ diameter roll and 1.07 $\mathrm{mm}$ for $68 \mathrm{~mm}$ diameter roll.

For measuring the distribution of curvature in a sheet, the sheet specimens are sheared $(280 \times 15 \mathrm{~mm})$ at 5 different positions along the width as shown in Fig. 12. The curvatures of each strip are calculated from the coordinate of 4points of the strip under non-restricted condition, standing the strip by sheared (thickness) face on the section paper above the non-magnetized surface table as suggested by Matoba. ${ }^{2)}$ The deviation of curvature in incoming and leveled strip is summarized in Fig. 13. Strip shape before leveling shows that curvature deviation in the sheet, occurred at the upstream, exist and the maximum curvature difference of strips within a sheet before leveling is about 0.7 




Fig. 13. Curvature distribution of strips sheared from sheets, incoming and leveled at A-Line roller leveler. Entrance intermesh value of roller leveler is about $5 \mathrm{~mm}$.

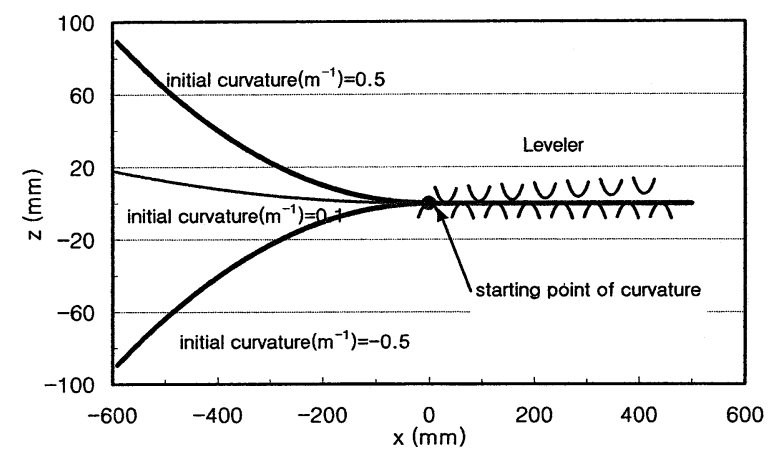

Fig. 14. Modeling of three levels of initial curvature.

Table 3. Mechanical properties of sheets used for finite element analysis and experiment.

\begin{tabular}{|c|c|c|c|c|c|c|}
\hline & $\begin{array}{c}\text { Thick. } \\
(\mathrm{mm})\end{array}$ & $\begin{array}{c}\text { YS } \\
(\mathrm{MPa})\end{array}$ & $\begin{array}{c}\text { YP-E1. } \\
(\boldsymbol{\%})\end{array}$ & $\begin{array}{c}\text { TS } \\
(\mathrm{MPa})\end{array}$ & $\begin{array}{c}\text { U-E1 } \\
(\boldsymbol{\%})\end{array}$ & $\begin{array}{c}\text { T-E1 } \\
(\boldsymbol{\%})\end{array}$ \\
\hline Sheet-1 & 0.21 & 405 & 6.3 & 432 & 15.3 & 24.6 \\
\hline Sheet-2 & 0.21 & 404 & 8.3 & 430 & 15.0 & 21.9 \\
\hline Sheet -3 & 0.21 & 398 & 7.8 & 428 & 14.5 & 22.7 \\
\hline Sheet-4 & 0.21 & 388 & 7.9 & 426 & 15.0 & 23.4 \\
\hline Average & 0.21 & 392 & 8.3 & 422 & 14.0 & 23.0 \\
\hline
\end{tabular}

$\mathrm{m}^{-1}$. The curvature deviation in a sheet is retained in some cases, even after the roller leveling.

Finite element simulation model for strips with initial curvature difference value of $1.0 \mathrm{~m}^{-1}$ are constructed by considering the strip shapes of incoming sheet. As described in Fig. 14, three specific curvature values of 0.5 $\mathrm{m}^{-1}, 0.1 \mathrm{~m}^{-1}$ and $-0.5 \mathrm{~m}^{-1}$ are chosen.

The material properties of considered sheet are summarized in Table 3. Due to large yield point elongation and small work hardening characteristics, flow curve is modeled as linear hardening material. Yield stress is $392 \mathrm{MPa}$ and plastic modulus $(H)$ is computed by yield stress $(Y S)$, true tensile stress $\left(T S_{\mathrm{t}}\right)$ and true plastic uniform elongation $\left(U E l_{\mathrm{pt}}\right)$ as following equation.

$$
H=\frac{T S_{\mathrm{t}}-Y S}{U E l_{\mathrm{pt}}}=688 \mathrm{MPa}
$$

Figure 15 presents simulation procedures of roller leveling for strip that had initial curvature value of $0.1 \mathrm{~m}^{-1}$ under $5 \mathrm{~mm}$ entrance and $0 \mathrm{~mm}$ exit intermesh at B-Line roller leveler. Initial curvature is assigned to part of sheet to be leveled under steady state deformation. Generally the

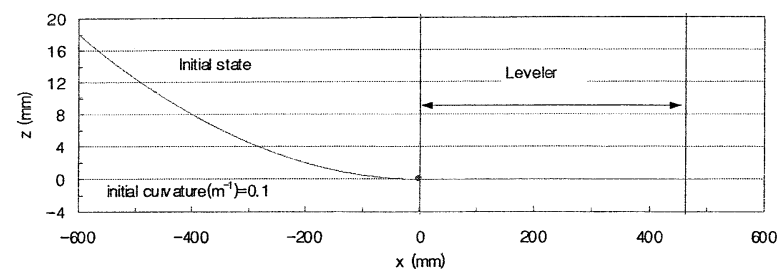

(a) Initial modeling for B-Line roller leveler

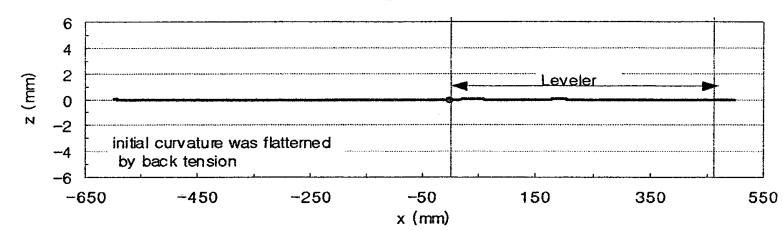

(b) Straightening of initial curvature

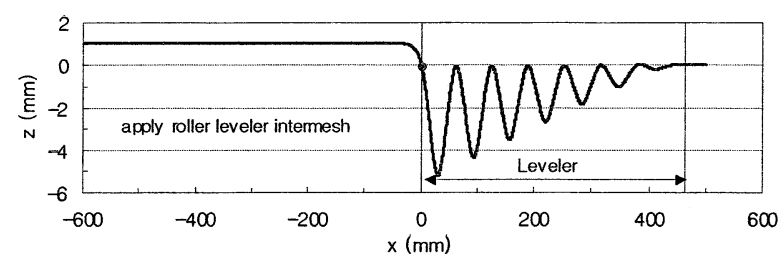

(c) Apply intermesh (Entrance. $=5 \mathrm{~mm}$, Exit $=0 \mathrm{~mm}$ )

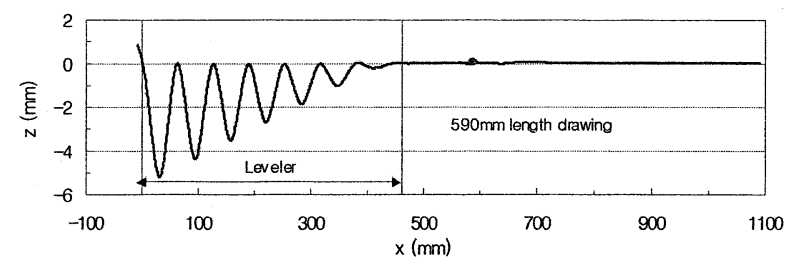

(d) Shape of sheet after 590mm length drawing.

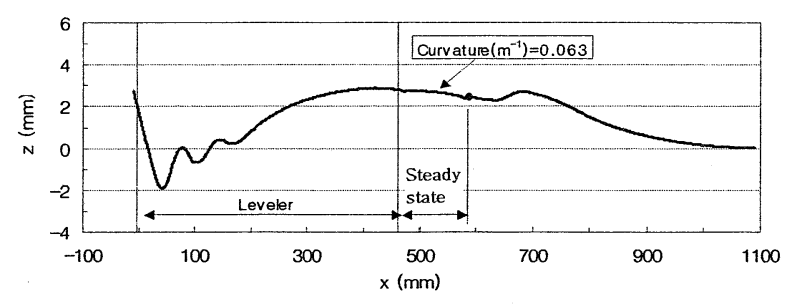

(e) after spring back

Fig. 15. Simulation procedure of roller leveling process.

strip before leveling is flattened to zero curvature due to the weight of sheet and constraint by processing roll arrangement, therefore incoming sheet should be flattened, though the sheet has some curvature in unconstrained condition. In this research, the initial curvature of strip is flattened by small tension, 19.6 MPa, which is enough for straightening initial curvature assumed here, and apply intermesh and level by drawing the strip. After $590 \mathrm{~mm}$ drawing, deformed length under steady state condition reached about $100 \mathrm{~mm}$, which is enough for evaluation of curvature after spring back, spring back analysis is performed.

\subsection{Analysis Results}

Figure 16 shows the deformation of a strip during leveling. Contact between the strip and roll is found to occur at an upstream point (before passing the vertical line drawn from the roll center), except the first roll where the point of contact is found at a downstream position, and maximum curvatures of sheet at contacting points are less than the curvature of roll due to the strip stiffness. Figure 17 shows the curvatures of sheet during leveling for two entrance in- 


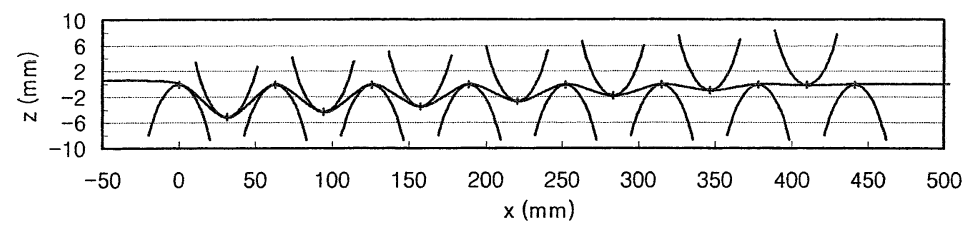

(a)

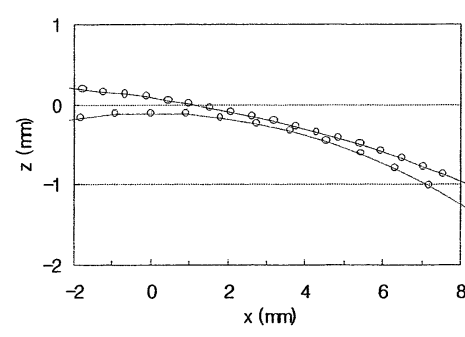

(b)

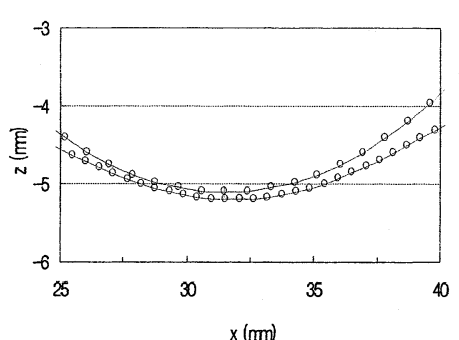

(c)

Fig. 16. Deformation and contact of sheet during leveling. ( $\mathrm{IM}=5 \mathrm{~mm}$, B-Line leveler)

(a) Deformation of strip and contact status with rolls

(b) Contact of sheet and the first roll

(c) Contact of sheet and the second roll

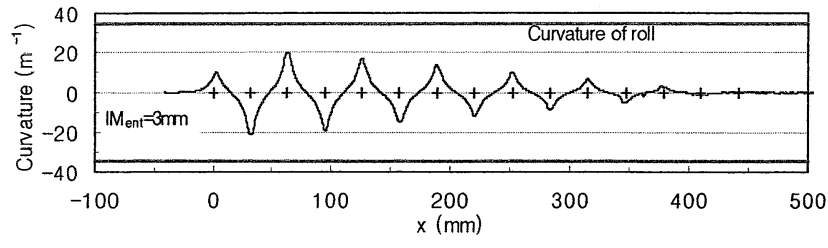

(a) Intermesh at entry side $=3 \mathrm{~mm}$

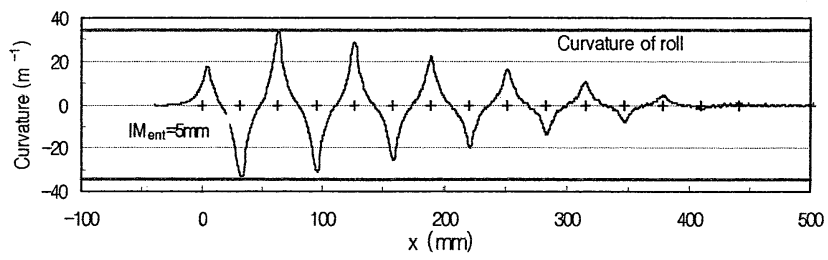

(b) Intermesh at entry side $=5 \mathrm{~mm}$

Fig. 17. Curvature of sheet at two entrance intermesh case for BLine leveler.

termesh conditions, (a) is $3 \mathrm{~mm}$ and (b) is $5 \mathrm{~mm}$ case. Curvatures of sheet are monotonically decreasing as sheet contact with rolls from entrance to exit of roller leveler. The curvature of sheet is less than the roll curvature, and it shows that sheet curvature was strongly affected by the leveler entrance intermesh.

Figure 18(a) presents the curvature of sheet after leveling with three different initial curvatures at A-Line roller leveler, its roll diameter is $68 \mathrm{~mm}$ and number of roll is 13 . The difference in residual curvature of sheet is reduced to zero when entrance intermesh was more than $6 \mathrm{~mm}$. Considering the average curvature of sheet and some safety factor, the suitable entrance intermesh value for removing blanking bow defects in A-Line roller leveler is $7 \mathrm{~mm}$. By comparing this results and the non-uniform curvature distribution of leveled sheet in Fig. 13, $5 \mathrm{~mm}$ entrance intermesh for A-Line leveler is small for removing non-uniform curvature in a sheet. This is the cause of blanking bow defects of sheet leveled in this roller leveler. After increasing the entrance intermesh of this leveler to $7 \mathrm{~mm}$, no blanking bow defects is claimed from customer. Figure 18(b) shows the

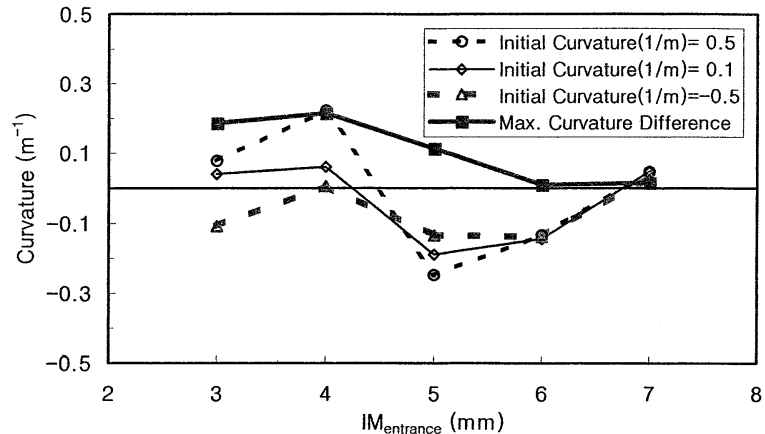

(a) A-Line roller leveler. (Number of rolls are 13 and diameter is $68 \mathrm{~mm}$. )

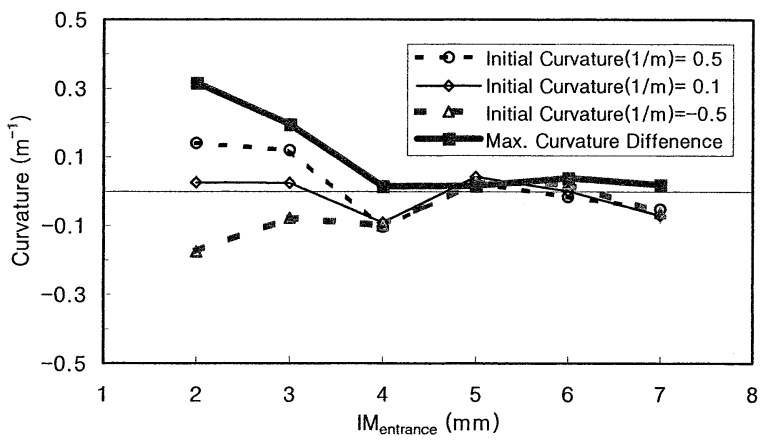

(b) B-Line roller leveler. (Number of rolls are 15 and diameter is $58 \mathrm{~mm}$.)

Fig. 18. Curvatures of leveled strips with different initial curvatures and the maximum curvature difference after leveling.

results of curvature of sheet after leveling for three different initial curvatures at B-type roller leveler, which has 15 rolls and diameter of each roll is $58 \mathrm{~mm}$. Suitable entrance intermesh for considered incoming sheet is predicted as $5 \mathrm{~mm}$. Figure 19 is the curvature distribution of incoming and leveled sheet at B-Line roller leveler at $6 \mathrm{~mm}$ entrance intermesh value. The non-uniform curvature of incoming sheet was leveled to the same curvature. By comparing with the simulated results in Fig. 18(b), experimental results shown in Fig. 19 could be explained and the entrance intermesh 




Fig. 19. Sheet curvature distribution before and after roller leveling. (B-Line roller leveler at $6 \mathrm{~mm}$ entrance intermesh values)

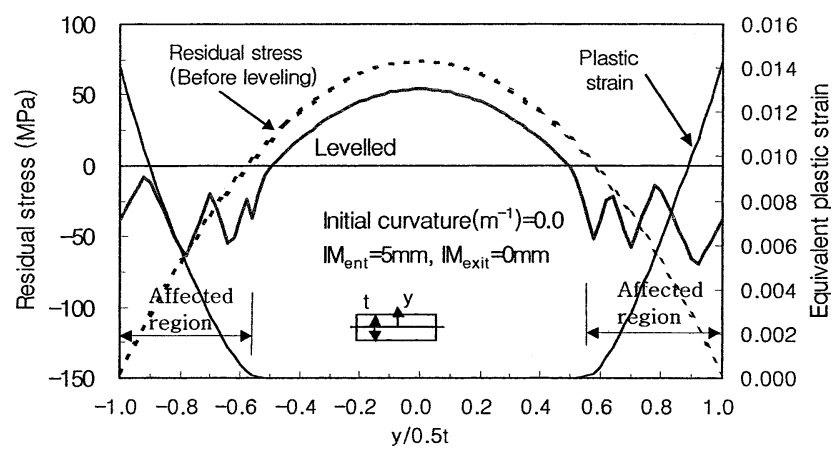

Fig. 20. Distribution of residual stress, before and after leveling, and equivalent plastic strain along thickness. (B-Line roller leveler, sheet thickness is $0.21 \mathrm{~mm}$.)

value used in this operation is sufficient for removing blanking bow defects.

\subsection{Prediction of Residual Stress and Comparison with Experiment}

From the results of finite element simulation of roller leveling, curvature of sheet at each roll is obtained. These curvatures of sheet are used for the numerical analysis of bending-unbending process and the residual stresses are predicted and compared with experimental results. This procedure is explained in Sec. 3 and almost the same as Kano ${ }^{10)}$ 's approach.

The measurement of the residual stress distributions in a strip revealed that the distributions are almost identical regardless of the position of the strip in the sheet before slitting, and that, as reported by Matoba, ${ }^{25)}$ they may be approximated by a parabolic curve, with $74 \mathrm{MPa}$ at the center and $-147 \mathrm{MPa}$ at the strip surface. Figure 20 is the distribution of the equivalent plastic strain and residual stress along thickness. Defining an affected region as the part where the fluctuation of the residual stress occurs, it may be seen from Fig. 20 that the affected region coincides with the plastically deformed zone. The result indicates that by measuring the residual stress distributions, the plastically deformed zone may be determined. The affected region is found to be increasing with the increase of the entrance intermesh, as shown in Fig. 21. It is also noted that with the increase of the affected region, the peak residual stress is decreasing, clearly indicating the effect of the entrance intermesh, not only on the blanking bow but also on the resid-

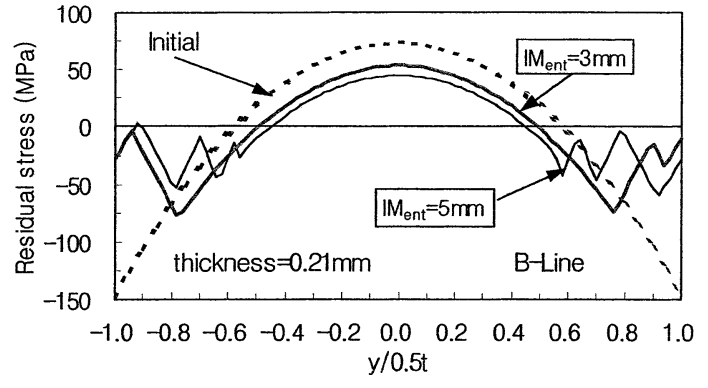

Fig. 21. The effects of entrance intermesh on the residual stress distribution of sheet after leveling. (B-Line leveler)

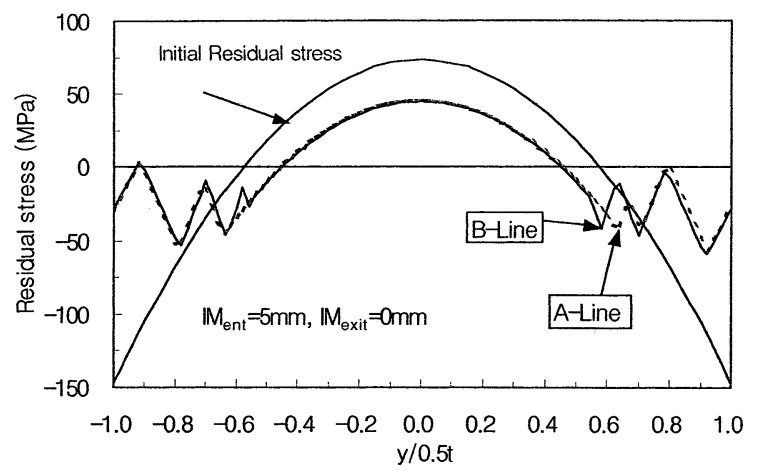

Fig. 22. The effects of roller leveler types on the residual stress distribution of sheet after leveling. Entrance intermesh value for both cases is $5 \mathrm{~mm}$.

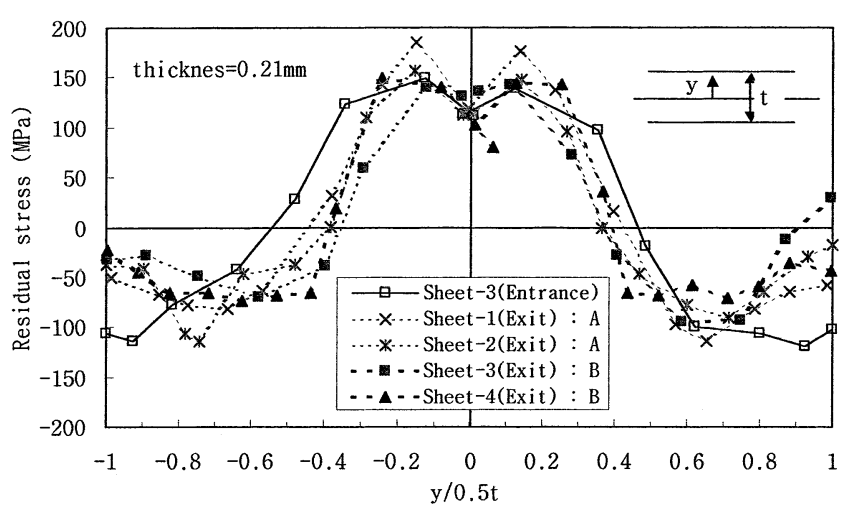

Fig. 23. Residual stress distributions of incoming and leveled sheet. Entrance intermesh of A-Line roller leveler was 5 $\mathrm{mm}$ and B-Line roller leveler was $6 \mathrm{~mm}$.

ual stress.

At the same entrance intermesh condition $(5 \mathrm{~mm})$ for two different levelers, the distributions of predicted residual stress are compared in Fig. 22. The affected region of residual stress is $35 \%$ of thickness for A-Line and $45 \%$ of thickness for B-Line leveler. It is found that the roll diameter is also an important factor on the leveler and the affected region of residual stress near surface was an important indication of leveling conditions. Figure 23 is the experimental results of residual stress distribution for $5 \mathrm{~mm}$ entrance intermesh for A-Line roller leveler and $6 \mathrm{~mm}$ entrance intermesh for B-Line roller leveler. For A-Line leveler, the plastically affected region of stress near surface is about $35 \%$ of thickness and $55 \%$ for B-Line leveler. The results show good agreement with simulated results shown in Fig. 22. 


\section{Conclusion}

For the analysis of roller leveling process and application to controlling blanking bow defects in thin steel sheets, a finite element analysis program modeling large deformation of shell element has been developed. This program was verified by the simple leveling experiment using 5 rolls at laboratory. The residual curvatures of strips predicted by finite element analysis were within $20 \%$ error range of the experimental results, and the residual stress distribution of leveled sheet was predicted, with reasonable accuracy, by using the curvature of sheet near the contacting point with rolls during leveling and numerical analysis of bending-unbending process.

Virtually divided strip model is applied in order to find the conditions for removing blanking bow defects. An optimum entrance intermesh condition, that could remove the deviation of curvature in incoming sheets, for two types of roller leveler are found by finite element simulation of initially curved sheet. The maximum difference in curvatures of incoming sheets at roller leveler is measured and reflected in the initial curvature of strip. The curvature difference of strips after leveling is decreased as increasing the entrance intermesh of roller leveler, and the predicted optimum intermesh value for removing blanking bow is affected by the roll diameter and arrangement. The experimental results are consistent with the optimum intermesh values obtained by applying virtually divided strip model for blanking bow. It is also found that the residual stress distributions after leveling are markedly affected by the intermesh condition. It is expected that, via more process simulation, a relation between the affected region of residual stress and the intermesh condition can be determined, which may also be used in tracing backward the intermesh condition that a sheet product has received.

\section{REFERENCES}

1) T. Masui: J. Jpn. Soc. Technol. Plast., 35 (1994), 94

2) T. Matoba, M. Ataka, I. Aoki and T. Jimma: J. Jpn. Soc. Technol.
Plast., 36 (1995), 1312.

3) Straightening of Metal Products-Technology to Straightening Sheet, Tube and Others-, ed. by Japan Society for Technology of Plasticity, Corona Publishing Co., Tokyo, (1992), 64.

4) Y. Misaka and K. Masui: J. Jpn. Soc. Technol. Plast., 17 (1976), 988. [English version in Trans. Iron Steel Inst. Jpn., 18 (1978), 477.]

5) S. Hattori, K. Maeda, T. Matsushita, S. Murakami and J. Hata: J. Jpn. Soc. Technol. Plast., 28 (1987), 34.

6) F. Hibino: J. Jpn. Soc. Technol. Plast., 31 (1990), 208.

7) T. Kajihara, H. Furumoto, T. Takemasa and N. Taniguchi: Tech. Rev. Mitsubishi Heavy Industry, 25 (1988), 1.

8) N. E. Thompson: Proc. of the 3rd Int. Conf. on Technologies of Plasticity, Vol. 3, ICTP, Kyoto, (1990), 1507.

9) F. Yoshida and M. Urabe: J. Mater. Process. Technol., 89-90 (1999), 218.

10) H. Kano, K. Kenmochi and I. Yarita: Proc. of the 7th Int. Conf. on Steel Rolling (Steel Rolling '98), ISIJ, Tokyo, (1998), 313.

11) T. Hira, H. Abe and S. Azuma: Kawasaki Steel Eng. Rep., 20 (1988), 7.

12) T. J. R. Hughes and W. K. Liu: Comput. Meth. Appl. Mech. Eng., 26 (1981), 331.

13) R. M. McMeeking and J. R. Rice: Int. J. Solids Structures, 11 (1975), 601.

14) J. Chakrabarty: Theory of Plasticity, McGraw-Hill International Editions, Singapore, (1987), 103.

15) A. S. Khan and S. Huang: Continuum Theory of Plasticity, John Wiley \& Sons, Inc., New York, (1995), 254.

16) J. C. Simo and T. J. R. Hughes: Computational Inelasticity, SpringerVerlag, Inc., New York, (1998), 266.

17) T. J. R. Hughes and J. Winget: Int. J. Numer. Meth. Eng., 15 (1980), 1862.

18) J. C. Simo and R. L. Taylor: Comput. Meth. Appl. Mech. Eng., 48 (1985), 101.

19) J. C. Simo and T. J. R. Hughes: Computational Inelasticity, SpringerVerlag, Inc., New York, (1998), 311.

20) D. J. Benson and J. O. Hallquist: Comput. Meth. Appl. Mech. Eng., 78 (1990), 141

21) Z. H. Zhong: Finite Element Procedures for Contact-Impact Problems, Oxford University Press, New York, (1993), 210.

22) Y. T. Keum, E. Nakamachi, R. H. Wagoner and J. K. Lee: Int. J. Numer. Meth. Eng., 30 (1990), 1471.

23) J. M. Roberts and T. Sheppard: J. Inst. Met., 99 (1971), 293.

24) T. Matoba, M. Ataka, Y. Sakamoto and F. Taki: Proc. of '86 Iron and Steel Cong., ISIJ, Tokyo, (1986), S381.

25) T. Matoba, K. Nagahiro and F. Taki: J. Jpn. Soc. Technol. Plast., 42 (2001), 959. 